\title{
Environment-Assisted Quantum Transport
}

\section{Citation}

Rebentrost, Patrick, Masoud Mohseni, Ivan Kassal, Seth Lloyd, and Alán Aspuru-Guzik. 2009. Environment-assisted quantum transport. New Journal of Physics 11(3): 033003.

\section{Published Version}

doi:10.1088/1367-2630/11/3/033003

\section{Permanent link}

http://nrs.harvard.edu/urn-3:HUL.InstRepos:4646014

\section{Terms of Use}

This article was downloaded from Harvard University's DASH repository, and is made available under the terms and conditions applicable to Open Access Policy Articles, as set forth at http:// nrs.harvard.edu/urn-3:HUL.InstRepos:dash.current.terms-of-use\#OAP

\section{Share Your Story}

The Harvard community has made this article openly available.

Please share how this access benefits you. Submit a story.

\section{Accessibility}




\title{
Environment-Assisted Quantum Transport
}

\author{
Patrick Rebentrost, ${ }^{1}$ Masoud Mohseni, ${ }^{1}$ Ivan Kassal, ${ }^{1}$ Seth Lloyd, ${ }^{2}$ and Alán Aspuru-Guzik ${ }^{1}$ \\ ${ }^{1}$ Department of Chemistry and Chemical Biology, Harvard University, 12 Oxford St., Cambridge, MA 02138 \\ ${ }^{2}$ Department of Mechanical Engineering, Massachusetts Institute of Technology, 77 Massachusetts Avenue, Cambridge MA 02139
}

(Dated: February 10, 2009)

\begin{abstract}
Transport phenomena at the nanoscale are of interest due to the presence of both quantum and classical behavior. In this work, we demonstrate that quantum transport efficiency can be enhanced by a dynamical interplay of the system Hamiltonian with pure dephasing induced by a fluctuating environment. This is in contrast to fully coherent hopping that leads to localization in disordered systems, and to highly incoherent transfer that is eventually suppressed by the quantum Zeno effect. We study these phenomena in the FennaMatthews-Olson protein complex as a prototype for larger photosynthetic energy transfer systems. We also show that disordered binary tree structures exhibit enhanced transport in the presence of dephasing.
\end{abstract}

PACS numbers: 03.65.Yz, 05.60.Gg, 71.35.-y, 03.67.-a

The dynamical behavior of a quantum system can be substantially affected by the interaction with a fluctuating environment. Noise and decoherence collapse the quantum wavefunction, and one might be lead to expect an inhibitory effect on, for example, quantum transport involving coherent hopping of a (quasi-) particle between localized sites. One of the most important classes of quantum transport is the energy transfer in molecular systems [1], for example in the chromophoric light-harvesting complexes [2, 3]. The role of the environment in chromophoric systems [4, 5, 6] and model geometries [7] has been widely studied. The HakenStrobl model is used to describe Markovian bath fluctuations [7, 8, 9]. Quantum transport can also be affected by the wellknown quantum localization [10, 11]. Energy mismatches in disordered materials lead to destructive interference of the wavefunction and subsequently to localization of the quantum particle. Specifically, it has been argued that quantum localization can seriously limit computational power and/or quantum walk properties in binary tree structures [12], where an exponential speed-up over a classical random walk can in principle be observed [13]. Generally, the overall effect of environment and static disorder is expected to be negative. However, as we demonstrate here, in a large variety of transport systems and under proper conditions, the interaction with the environment can result in increased quantum transport efficiency.

In chromophoric complexes, an environment-assisted quantum walk approach within a Redfield model involving relaxation and dephasing was suggested to explain the high energy transfer efficiency [14]. This approach was also used to quantify the percentage contributions of quantum coherence and environment-induced relaxation to the overall efficiency [15]. Phonon-enhanced transfer has recently been reported for two quantum dots within the Redfield theory including relaxation [16]. Measurement of a single site in a quantum-dot array leads to complete delocalization of electrons in the onedimensional Anderson model [17]. Noise-induced enhancement can also been seen in stochastic resonance [18], where the quantum system is driven to a non-linear regime. The role of pure dephasing in quantum localization was discussed in [19, 20, 21, 22]. Several authors investigated quantum entanglement in biological systems in the presence of dissipa-

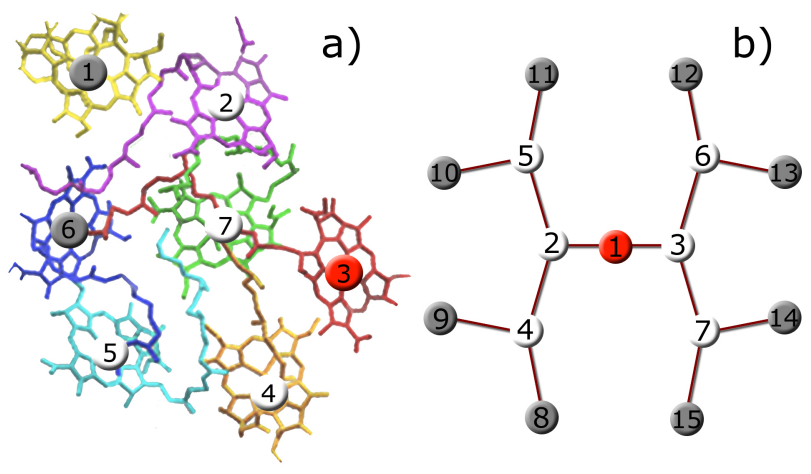

FIG. 1: Quantum transport occurs in natural and engineered systems, for example: a) Energy transfer in photosynthetic complexes between chlorophyll molecules, such as the Fenna-Matthews-Olson protein complex, in which quantum coherence has been shown to play a significant role in the exciton dynamics [2]. (b) Transport of particles and/or information in artificial or engineered systems described by a tight-binding Hamiltonian. Here, a four generation binary tree is shown. In particular, an exponential speed-up in reaching certain target sites (red) in these structures has been proposed in the context of quantum walk algorithms [13]. (The grey sites represent initial states for the quantum transport.)

tive dynamics, reset mechanisms, and molecular oscillations [23, 24].

Interactions of a quantum system with a thermal environment or measurement of an observable can lead to the Zeno and anti-Zeno effects. In the Zeno effect [25], sometimes also termed the 'watchdog effect', repeated, fast measurement suppresses the free evolution governed by Schrödinger's equation. The quantum system remains in an eigenstate of the measurement operator. The anti-Zeno effect describes the opposite scenario [26, 27, 28]. Here, such interactions, if well timed, accelerate certain processes, such as the decay of an unstable state, compared to the unperturbed case.

In this work, we study the interaction between pure dephasing noise and coherent dynamics that can result in greatly increased efficiency of transport. Unlike stochastic resonance, this enhancement occurs in undriven systems. The intuition is 
as follows: In a quantum system with some degree of disorder, localization suppresses transport at low noise levels. By contrast, at very high noise levels, decoherence effectively produces a 'watchdog effect' that also suppresses transport. However, at intermediate noise levels coherence and decoherence can collaborate to produce highly efficient transport. This enhancement holds even if the noise itself is purely decohering and can induce no transport on its own. We investigate a general dephasing model of such environment-assisted quantum transport (ENAQT) and apply that model to the FennaMatthews-Olson complex and binary trees. For the FennaMatthews-Olson complex we note that the pure-dephasing model is highly idealized, since it ignores exciton relaxation and temporal/spatial correlations in the environment. Within these limitations, we show that the interplay between coherence and decoherence leads to maximally efficient transport at a noise level corresponding to ambient temperature.

Master equations for quantum transport. The tight-binding Hamiltonian for an interacting $N$-body system in the presence of a single excitation is given by [1]:

$$
H_{\mathrm{S}}=\sum_{m=1}^{N} \epsilon_{m}|m\rangle\langle m|+\sum_{n<m}^{N} V_{m n}(|m\rangle\langle n|+| n\rangle\langle m|) .
$$

This Hamiltonian applies to a large class of quantum transport systems such as excitons and charges in molecular crystals and quantum dots [29, 30]. The states $|m\rangle$ denote the excitation being at site $m$. The site energies and two-body interactions are given by $\epsilon_{m}$ and $V_{m n}$ respectively. The site energies, or static disorder, can be due to different local environments of otherwise identical molecules or due to fabrication imperfections of engineered structures. For chromophores, the coupling is mediated by the Coulomb interaction (Förster coupling) or electron exchange (Dexter coupling). It is appropriate to study the dynamics only in the single exciton manifold, spanned by the states $|m\rangle$. This is because in the absence of light-matter interaction events the number of excitons is conserved within the exciton recombination time scale of $1 \mathrm{~ns}$ [31], which is much longer than the usual time scales of the Hamiltonian (1) [14, 32, 33].

A multichromophoric system interacts with the surrounding environment, such as the solvent or the protein, which is usually a macroscopic system with many degrees of freedom. This coupling leads to irreversible dynamics which is characterized by relaxation of an exciton from a high- to a lowenergy state and dephasing of coherences. At ambient temperature and in common photosynthetic complexes the energy relaxation of an exciton occurs on a time scale of $\sim 1 \mathrm{ps}$, while dephasing occurs on a time scale of $\sim 100 \mathrm{fs}$ [9]. Thermal fluctuations of the environment couple to the chromophores by the electron-phonon Hamiltonian:

$$
H_{\mathrm{SB}}(t)=\sum_{m} q_{m}(t)|m\rangle\langle m|,
$$

where the $q_{m}(t)$ describe stochastic bath fluctuations. Here, we consider only diagonal fluctuations which are typically larger than fluctuations of the inter-molecular couplings [29. 33]. To a certain approximation, the decoherence part of the resulting equation of motion for a multi-level system in the presence of Markovian fluctuations is dominated by pure dephasing [8, 9]. This is especially the case at high temperatures. The Liouville-von Neumann equation for the system when averaging over the fluctuations is $\dot{\rho}(t)=-\frac{i}{\hbar}\left\langle\left[H_{\mathrm{S}}+\right.\right.$ $\left.\left.H_{\mathrm{SB}}(t), \rho(t)\right]\right\rangle$. The random variables $q_{m}(t)$ are taken to be unbiased Gaussian fluctuations, with $\left\langle q_{m}(t)\right\rangle=0$ and a twopoint correlation function [9, 29, 33]:

$$
\left\langle q_{m}(t) q_{n}(0)\right\rangle=\delta_{m n} \delta(t) \gamma_{\phi},
$$

where $\gamma_{m}$ is a site-dependent rate. First, we assumed that fluctuations at different sites are uncorrelated. Second, we assumed that the phonon correlation time is small compared to the system timescales, an assumption that is justified at room temperatures where the phonon correlation time is estimated to be below 50 fs [9]. Finally, the correlator is assumed to be site-independent, so all chromophores experience the same coupling strength to the environment, $\gamma_{\phi}$. With these assumptions, one obtains the Haken-Strobl equation for the density operator in the Schrödinger picture as [8]:

$$
\dot{\rho}(t)=-\frac{i}{\hbar}\left[H_{\mathrm{S}}, \rho(t)\right]+L_{\phi}(\rho(t)),
$$

where the pure-dephasing Lindblad operator is given by:

$$
L_{\phi}(\rho(t))=\gamma_{\phi} \sum_{m}\left[A_{m} \rho(t) A_{m}^{\dagger}-\frac{1}{2} A_{m} A_{m}^{\dagger} \rho(t)-\frac{1}{2} \rho(t) A_{m} A_{m}^{\dagger}\right] .
$$

with the generators $A_{m}=|m\rangle\langle m|$ and a pure dephasing rate is given by $\gamma_{\phi}$. This Lindblad equation leads to exponential decay of all coherences in the density operator.

Energy transfer efficiency and transport time. There are several possible ways to measure or quantify the success rate of an energy transfer process, such as energy transfer efficiency and transfer time [32, 34, 35, 36]. First, in order to account for exciton recombination and exciton trapping, we augment the Hamiltonian (1) with anti-Hermitian parts [14, 37],

$$
\begin{aligned}
H_{\text {recomb }} & =-i \hbar \Gamma \sum_{m}|m\rangle\langle m|, \\
H_{\text {trap }} & =-i \hbar \sum_{m} \kappa_{m}|m\rangle\langle m| .
\end{aligned}
$$

The exciton recombines with a rate $\Gamma$ at every site and is trapped with a rate $\kappa_{m}$ at certain molecules [7, 9, 34, 38]. The probability that the exciton is successfully captured at a target site $m$ within the time interval $[t, t+d t]$ is given by $2 \kappa_{m}\langle m|\rho(t)| m\rangle d t$. Thus, the efficiency can be defined as the integrated probability of trapping at multiple sites as:

$$
\eta=2 \sum_{m} \kappa_{m} \int_{0}^{\infty} d t\langle m|\rho(t)| m\rangle .
$$

In general, the efficiency is reduced by finite exciton lifetimes $(\sim 1 \mathrm{~ns})$. Another relevant measure for a quantum transport process is the average transfer time defined as:

$$
\tau=\frac{2}{\eta} \sum_{m} \kappa_{m} \int_{0}^{\infty} d t t\langle m|\rho(t)| m\rangle .
$$


The efficiency of quantum transport elucidates the shorter time scales given by the trapping/recombination rates. This approach differs from approaches that consider the limiting distribution of site populations [13]. For example, the limiting distribution of a pure-dephasing master equation, such as Eq. (4) (i.e. without the trapping/recombination part), is an equal population of all sites, which is the same as for a classical random walk on a regular graph [13]. The efficiency captures the physically relevant shorter time scales.

Environment-assisted quantum transport (ENAQT). The efficiency of quantum transport in an open system can be substantially enhanced by the interaction with a fluctuating environment. The master equation (4) is a specific example of a large class of transport master equations representing siteto-site hopping situations. As noted above, the Hamiltonian part of these master equations has a diagonal part representing the energies of the individual sites, while the off-diagonal part represents hopping terms. The open-system Lindblad operators in the master equation are dominated by terms that dephase the system in the site basis. Relaxation, another possible non-unitary contribution to the quantum transport involving energy exchange with the environment, is rather slow compared with dephasing. The dephasing rate is slow at low temperatures, and fast at high temperatures. Based on fundamental physical principles, we can make the following set of phenomenological predictions for such decohered quantum evolution. As will be seen, these predictions are borne out by the simulated behavior of the Fenna-Matthews-Olson complex and in binary trees. We predict that the same generic behavior will hold for decohered quantum walks in general.

At low temperatures, the dynamics is dominated by coherent hopping. Because of the variation in the energy levels of different sites and in the strength of the hopping terms, the system is disordered and exhibits quantum localization [10]. The degree of localization depends on the variation in the energies: for small variation, the system should exhibit weak localization, and for large variation, strong localization should take place. Note that the characteristic behavior of quantum localization can occur even in a system with only a few sites [39]. In this case, localization can be thought of in terms of energy conservation: an excitonic state originally localized at an initial site is a superposition of energy eigenstates that exhibits only a slight overlap with an excitonic state localized at a final state with significantly different site energy. As a result, coherent hopping on its own has a low efficiency for transporting an excitation from one site to another with significantly different site energy.

As the temperature rises, dephasing comes into play. At first, it might seem that dephasing in the site basis can have no role in enhancing transport, as this form of noise induces no transport on its own. A moment's reflection, however, reveals that this expectation is incorrect. Localization is caused by coherent interference between paths; if that coherence is destroyed, then the localization effect is mitigated. Coherence causes an excitation to become 'stuck.' It might oscillate back and forth between a few sites that are strongly coupled and have similar energies, but the exciton will never venture far afield. By destroying the coherence of the beating, dephasing also destroys the localization and allows the exciton to propagate through the system. This phenomenon, by which decoherence enhances transport, affects all such hopping systems.

When the dephasing rate grows larger than the terms of the system Hamiltonian we expect transport to be suppressed again. This suppression of transport by high dephasing can be thought of as an example of the watchdog (quantum Zeno) effect: rapid dephasing at a rate $\gamma_{\phi}$ in the site basis is equivalent, so far as the system is concerned, to being measured repeatedly in the site basis at time intervals $\approx \gamma_{\phi}^{-1}$. The watchdog effect will then suppress transport away from the initial site. While these predictions hold for a large class of transport systems, we study these effects for three different systems including a two-chromophore system, the Fenna-Matthews-Olson complex, and a binary tree structure.

Quantum transport in a two chromophore system. A particularly simple and illustrative example is to study quantum transport in a system of two sites without trapping and recombination: a particle hops from site 1 to 2 with a significant energy mismatch between 1 and 2 . With $|1\rangle$ and $|2\rangle$, the states where the exciton is localized at site 1 and 2, respectively, the Hamiltonian for such a system can be written $H=\epsilon / 2(|1\rangle\langle 1|-| 2\rangle\langle 2|)+V / 2(|1\rangle\langle 2|+| 2\rangle\langle 1|)$, where $\epsilon$ is the energy mismatch between 1 and 2 , and $V$ is the strength of the hopping term. As usual, we define the Larmor frequency $\hbar \Omega=\sqrt{\epsilon^{2}+V^{2}}$. The coherent evolution of the system, starting from site 1 , is simply a rotation about an axis displaced by an angle $\theta=\sin ^{-1}(V / \hbar \Omega)$ from the $z$-axis in the $x-z$ plane. The maximum probability of finding the system at site 2 is $\sin ^{2} 2 \theta$, and the average probability of finding it there is $\sin ^{2} \theta$. If the energy mismatch is sufficiently large, substantial hopping does not occur and the system remains localized at site 1.

In the presence of decoherence, the system obeys the Bloch equation. Pure dephasing corresponds to a Lindblad operator $\sqrt{\gamma_{\phi}}(|1\rangle\langle 1|-| 2\rangle\langle 2|)$, where $\gamma_{\phi}=1 / T_{\phi}$. The conventional Bloch analysis now holds. The system, instead of remaining localized at site 1, gradually diffuses, ultimately becoming a uniform mixture of $|1\rangle$ and $|2\rangle$. In the equilibrium state the system has a $50 \%$ chance of being found at site 2 . The diffusion process can be thought of as a random walk on the Bloch sphere with step length $\theta$ and with time per step $\gamma_{\phi}^{-1}$. Accordingly, the system must perform $\approx(\pi / \theta)^{2}$ steps and the diffusion time is $\tau_{\text {diff }} \approx(\pi / \theta)^{2} \gamma_{\phi}^{-1}$ to reach a steady state. For a system with more than two sites, the transport will be more complicated. Nevertheless, we still expect the transport rate to increase in direct proportion to the inverse of the individual site decoherence time. This is indeed true if the decoherence time does not substantially exceed the time scales defined by the transport terms in the Hamiltonian and the energy mismatch from site to site. This fact supports the second prediction of environment-assisted quantum transport.

In the case of rapid dephasing, $\gamma_{\phi}>\Omega$, the angle $\phi$ that the system precesses before being decohered is $\approx \Omega / \gamma_{\phi}$. The probability of remaining in site 1 becomes $\cos ^{2} \phi \approx$ $1-\left(\Omega / \gamma_{\phi}\right)^{2}$. The system essentially performs a biased random walk with step size $\phi$ and an average time per step of $\left(\gamma_{\phi} / \Omega\right)^{2} \gamma_{\phi}^{-1}=\gamma_{\phi} / \Omega^{2}$. In time $t$, the system diffuses by an 
angle $\Omega \sqrt{t / \gamma_{\phi}} \cdot \Omega / \gamma_{\phi}=\Omega^{2} t^{1 / 2} \gamma_{\phi}^{-3 / 2}$. In the case that the system has more than two states, we still expect this analysis to hold, taking $\gamma_{\phi}$ to be the dephasing rate and $\Omega$ to be an average eigenfrequency. This supports our third prediction: as the dephasing rate grows larger than the Hamiltonian energy scale, the transport rate is suppressed by a polynomial in the dephasing rate. The system will obviously converge to the same statistical mixture as mentioned above, albeit on a long and in some cases physically irrelevant time scale. These general properties of ENAQT are also observed by the simulations of the FMO complex and binary trees, where we include recombination and trapping.

Quantum transport in Fenna-Matthews-Olson protein complex. The Fenna-Matthews-Olson protein of the green sulphur bacterium Chlorobium tepidum [2, 29, 40] is a trimer in which each of the three subunits has seven chlorophyll molecules spatially arranged within a distance of several nm [41]. The three subunits can be treated independently from each other. The FMO complex transfers excitation energy from the chlorosomes, the main light-harvesting antennae, to a reaction center where a charge separation event and subsequent biochemical reactions occur. In analogy to the two-level system discussed above, we expect the same environmentassisted dynamical behavior in the FMO complex monomers. The dynamics of a single excitation is governed by a Hamiltonian of the form Eq. (1) for seven sites with a distribution of site energies and inter-site couplings as given in Ref. [29]. The chromophoric Förster couplings are up to $100 \mathrm{~cm}^{-1}$. The chlorophyll transition frequencies are shifted by the electrostatic protein environment, resulting in site-dependent electrochromic shifts of up to $300 \mathrm{~cm}^{-1}$ [33, 40]. Fluctuations of the protein in the solvent lead to fluctuations of the transition frequency of the chlorophyll molecules and therefore to loss of excitonic phase coherence. We use the master Eq. (4) with a site-independent dephasing rate $\gamma_{\phi}$ according to the Haken-Strobl model. This approach is not the standard method for describing decoherence effects within the FMO complex. Usually, relaxation [14, 29, 33] and spatial correlations [33] are included. In fact, a more complete description would also involve a phonon bath with memory effects. However, the Haken-Strobl model has already been used to describe the quantum dynamics of certain chromophoric arrays [7. 9] and is expected to deliver insight into the high temperature behavior of the FMO protein.

The initial state for our simulation of the system is a statistical mixture of localized excitations at sites 1 and 6, the chlorophyll molecules that are close to the chlorosome antenna. In the FMO complex, chromophore 3 is in the vicinity of the reaction center [33, 40, 41]. Thus, one can assume that chlorophyll 3 is the main excitation donor to the reaction center [14]. The precise transfer rate to the reaction center is not fully characterized. Yet, based on typical transfer rates in chromophoric complexes with similar inter-molecular distances, we estimate it to be $\kappa_{3}=1 \mathrm{ps}^{-1}$ [14]. Thus, the efficiency of energy transfer according to Eq. 8 becomes $\eta=2 \kappa_{3} \int_{0}^{\infty} d t\langle 3|\rho(t)| 3\rangle$.

In Fig. 2 (upper panel) the efficiency of transfer and the transfer time is given as a function of the dephasing rate $\gamma_{\phi}$. At low dephasing, purely quantum mechanical evolution leads
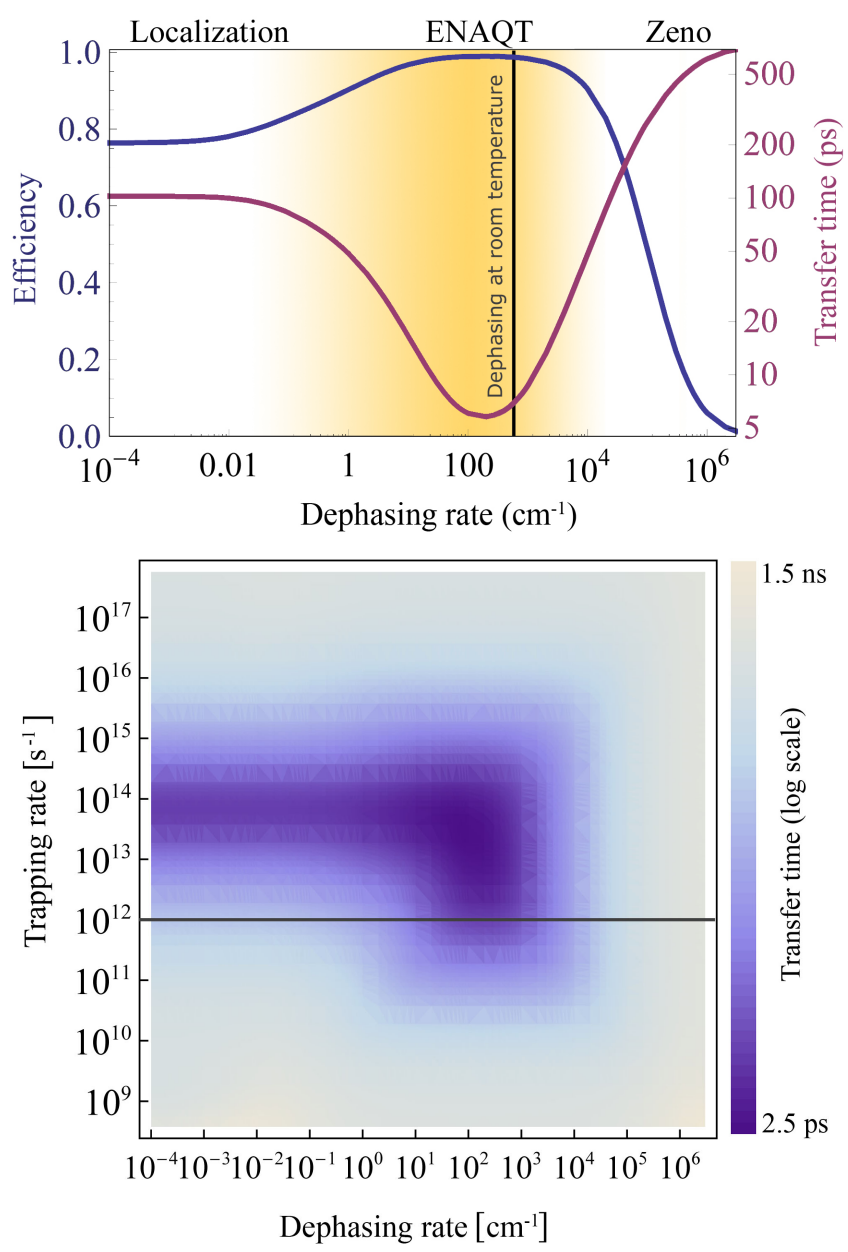

FIG. 2: (Upper panel) Efficiency (blue) and transfer time (red) as a function of the pure-dephasing rate is demonstrated for the FennaMatthew-Olsen complex. A clear picture of the three dephasing regimes is obtained: from left to right, the fully quantum regime which is dominated by intrinsic static disorder in the system Hamiltonian; the ENAQT regime (qualitatively indicated by the yellow color gradient), where unitary evolution and dephasing collaborate with the result of increased efficiency; finally, the quantum Zeno regime, where strong dephasing suppresses the quantum transport. As a guide to the eye, the estimated dephasing rate at room temperature for the FMO complex spectral density (see text) is drawn. The trapping rate is $\kappa_{3}=1 \mathrm{ps}^{-1}$. (Lower panel) Transfer time as a function of dephasing rate and trapping rate $\kappa_{3}$ is illustrated on a log scale. The upper panel is indicated as a horizontal line.

to an efficiency of around $80 \%$. With increasing dephasing the efficiency increases considerably, up to $94 \%$, where it approximately remains constant for a range of $\gamma_{\phi}$ of one order of magnitude. For stronger dephasing the efficiency is slowly suppressed again, delocalization is destroyed, and the overlap with the target site vanishes. The transfer time is $75 \mathrm{ps}$ in the fully quantum limit and improves significantly to $7 \mathrm{ps}$ in the intermediate ENAQT regime. For large dephasing, the transfer slows down to $500 \mathrm{ps}$, the same order of magnitude as the excitation lifetime: the exciton is more likely to recombine 
than to be trapped.

One can estimate the dephasing rate as a function of temperature by employing a standard system-reservoir model [42]. In this context, the spectral density is given by $J(\omega)=\sum_{i} \omega_{i}^{2} \lambda_{i}^{2} \delta\left(\omega-\omega_{i}\right)$, where $\omega_{i}$ are frequencies of the harmonic-oscillator bath modes and $\lambda_{i}$ are dimensionless couplings to the respective modes. In the continuum limit, we assume an Ohmic spectral density with cutoff, $J(\omega)=$ $\frac{E_{R}}{\hbar \omega_{c}} \omega \exp \left(-\omega / \omega_{c}\right)$. For the FMO complex, the reorganization energy is found to be $E_{\mathrm{R}}=35 \mathrm{~cm}^{-1}$ [29] and the cutoff $\omega_{c}=150 \mathrm{~cm}^{-1}$, inferred from Fig. 2 in Ref. [33]. In the Markovian regime, the dephasing rate $\gamma_{\phi}$ is given as the zero-frequency limit of the Fourier transform of the bath correlator. As a result, $\gamma_{\phi}$ is found to be proportional to the temperature and the derivative of the spectral density at vanishing frequency, $\gamma_{\phi}(T)=\left.2 \pi \frac{k T}{\hbar} \frac{\partial J(\omega)}{\partial \omega}\right|_{\omega=0}$ [6, 42]. For the above spectral density the rate turns out to be $\gamma_{\phi}(T)=2 \pi \frac{k T}{\hbar} \frac{E_{\mathrm{R}}}{\hbar \omega_{c}}$. This gives a rough estimate for the dephasing rate at room temperature of around $300 \mathrm{~cm}^{-1}$, which is indicated in Fig. 2 . Hence, the natural operating point of the FMO complex is estimated to be well within the regime of ENAQT, where the dephasing introduced by a fluctuating environment enhances the energy transfer efficiency. In Fig. 2 (lower panel), the transfer time is shown as a function of dephasing rate and trapping time $\kappa_{3}$. An optimal region with respect to dephasing rate and trapping rate is obtained.

Quantum transport in binary tree structures. Binary trees appear in a wide variety of situations, ranging from computer science [43] to quantum physics and quantum information science [13]. Specifically, they arise in classical and quantum random walks [13] and certain molecular structures such as dendrimers [44]. The effect of static disorder of the site energies in quantum walks on binary trees has been studied in Refs. [12, 35] where it was argued that such disorder diminishes the exponential speed-up in finding particular target sites, a consequence of quantum localization. The presence of disorder could restrict the applicability of binary tree structures for devising quantum algorithms or for transporting quantum particles. In this section, we demonstrate that ENAQT also occurs in a statically disordered binary tree structure leading to a substantial improvement in quantum transport.

The Hamiltonian of a disordered graph of generation $g$ is:

$$
\begin{aligned}
H_{\mathrm{S}}= & \sum_{m=1}^{2^{g}-1} \epsilon_{m}|m\rangle\langle m| \\
& +V \sum_{m=1}^{2^{g-1}-1}(|m\rangle\langle 2 m|+| m\rangle\langle 2 m+1|+\text { h.c. }) .
\end{aligned}
$$

The site energies $\epsilon_{m}$ are taken to be normally distributed about a common value $\epsilon_{0}$, where the standard deviation of the distribution, $\delta$, is the characteristic parameter of the static disorder. The hopping strength $V$ is uniform over the full graph and connects the sites as depicted in Fig. 1p for a four-generation graph (15 sites). In the presence of static disorder, the full Hilbert space has to be taken into account and consequently a reduction to a quantum walk on the line, as in [12, 13], is not possible. Additionally, we include exciton recombination at all the sites and exciton trapping at the center site 1. Both effects are again modeled by the anti-Hermitian Hamiltonians $H_{\text {recomb }}=-i \hbar \Gamma \sum_{m=1}^{2^{g}-1}|m\rangle\langle m|$ and $H_{\text {trap }}=-i \hbar \kappa|1\rangle\langle 1|$, with the recombination rate $\Gamma$ and the trapping rate $\kappa$. This defines the efficiency of transfer, Eq. (8). Dephasing is taken to be uniform over the whole graph according to the master equation 4 . We assume an initial state where all the sites in the outermost branch are populated in a classical mixture or a coherent superposition.

Fig. 3 shows the dependence of the transport efficiency on the characteristic disorder parameter $\delta$ for a fourth-generation binary tree, initially in a coherent superposition (left) or a statistical mixture (right). The parameters are $\Gamma=0.005 \mathrm{~V}$ and $\kappa=2 V$. For a given $\delta$ the efficiencies are calculated for 100 randomized graphs. The purely quantum case (blue) is compared to the case where the introduction of dephasing leads to an optimal enhancement of the energy transfer efficiency (orange). In the second case the efficiency of transport for each statically disordered graph was numerically maximized as a function the pure dephasing rate. In the figure, the broadening around the average efficiency indicates one standard deviation. For the initial state being a coherent superposition of all sites in the outermost branch, one can clearly see that static disorder leads to a reduced transport efficiency. This is readily explained by localization, see discussion above and Ref. [12]. Dephasing leads to an average improvement of the transport efficiency for the binary trees considered here. The average improvement is larger the more static disorder is in the system.

The behavior in the case of small static disorder changes when a classical mixture of excitations in the outermost branch is taken as an initial state. Since the initial state is not a column eigenstate that preserves the symmetry of the graph, the quantum limit without static disorder shows only small transport efficiencies of $20 \%$. Additional static disorder on average increases the efficiency to a maximum of $60 \%$ when $\delta / V \approx 1$. Here, static disorder creates higher localization at the root of the tree at site 1 . For larger static disorder one obtains the same suppression of the efficiency as for the coherent initial state. In the presence of environmental fluctuations one obtains an overall average improvement for all static disorder regimes: for small static disorder the improvement is $60 \%$, for intermediate it is $20 \%$, and for larger disorder it is around $40 \%$. In summary, ENAQT is shown to consistently improve the transport efficiency in binary tree structures, overcoming localization induced by static disorder of the site energies.

Conclusion. Environment-assisted quantum transport is a fundamental effect which occurs in a wide variety of transport systems. ENAQT is similar in flavor to stochastic resonance [18]: adding noise to a coherent system enhances a suitable transition rate. ENAQT differs from stochastic resonance in that the system whose transition rate is enhanced is undriven and does not need to be in some strong nonlinear regime. The maximum efficiency of ENAQT occurs when the decoherence rate is comparable to the energy scales of the coherent system as defined by the energy mismatch between states and the hop- 

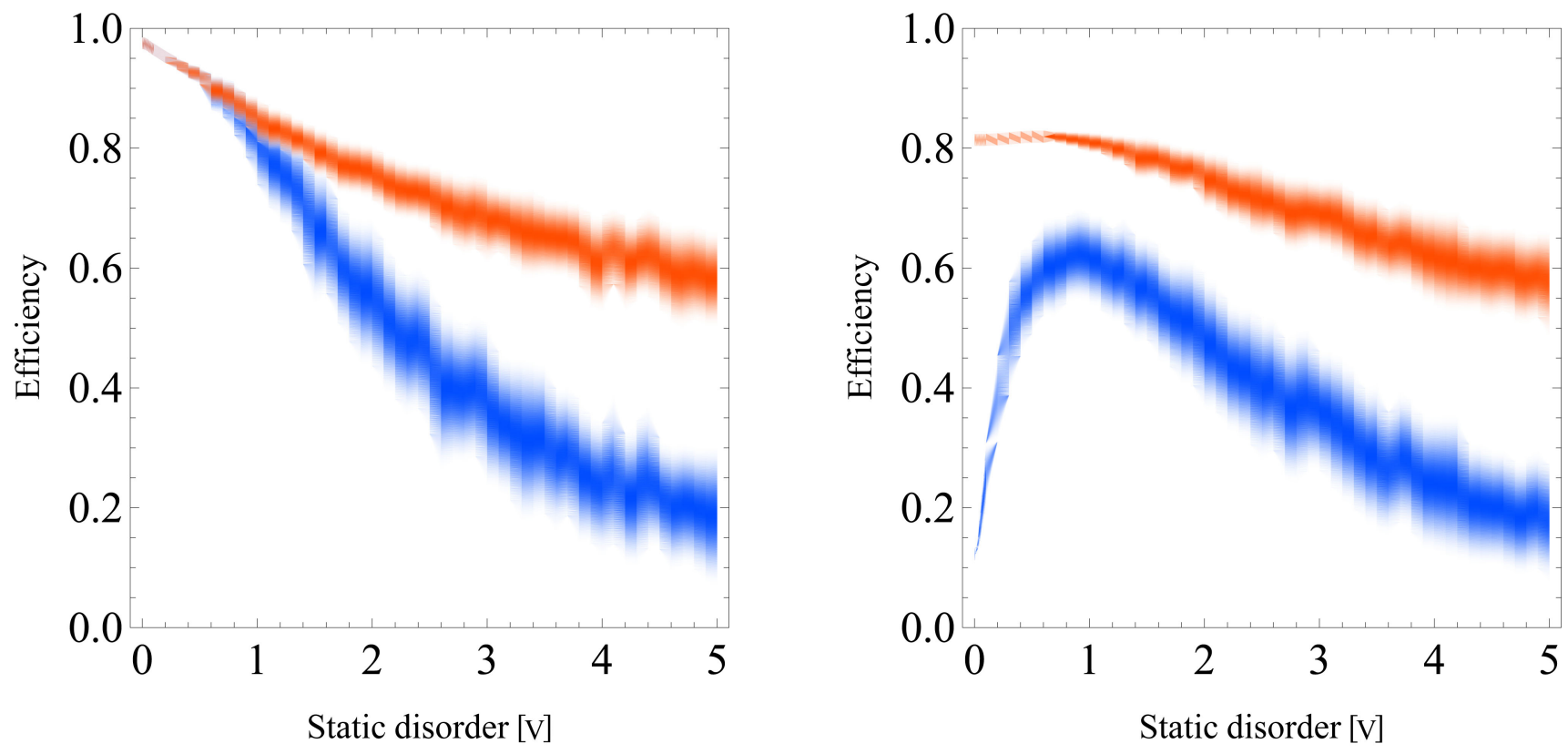

FIG. 3: The efficiency as a function of the static disorder parameter $\delta$ (in units of the coupling $V$ ) for a four-generation binary tree. A coherent superposition (left panel) or a statistical mixture (right panel) of the outermost branch of the tree was chosen as an initial state (cf. Fig. 1b, grey sites). In the fully quantum case (blue) large disorder reduces the efficiency due to quantum localization. 100 randomly sampled graphs were used per data point, leading to a distribution of efficiencies. For each statically disordered binary tree the optimal dephasing rate was calculated. The transport efficiency in the presence of dephasing with this optimal rate is considerably higher (orange). Parameters are $\Gamma=0.005 \mathrm{~V}$ and $\kappa=2 V$.

ping terms. By changing the energy mismatch and the hopping terms, the temperature at which the maximum transport efficiency occurs can be tuned. In the Fenna-Matthews-Olson protein complex within the pure dephasing model and with the spectral density as discussed above, this maximum occurs at approximately room temperature. Recently, results along the lines of this work were presented in [45]. Further studies are in order that utilize more advanced, non-Markovian decoherence models and detailed quantum chemistry calculations.

We would like to acknowledge useful discussions with G.R. Fleming and C.A. Rodriguez-Rosario. We thank the Faculty of Arts and Sciences of Harvard University, the Army Research Office (project W911NF-07-1-0304), and Harvard's Initiative for Quantum Science and Engineering for funding.
[1] V. May and O. Kühn, Charge and Energy Transfer Dynamics in Molecular Systems (Wiley-VCH, Weinheim, 2004).

[2] G.S. Engel, T.R. Calhoun, E.L. Read, T.-K. Ahn, T. Mancal, Y-C. Cheng, R.E. Blankenship, and G.R. Fleming, Nature 446, 782 (2007).

[3] H. Lee, Y.-C. Cheng, and G.R. Fleming, Science 316, 1462 (2007).

[4] M. Grover, R. Silbey, J. Chem. Phys. 54, 4843 (1971).

[5] M. Yang, G.R. Fleming, Chem. Phys. 275, 355 (2002).

[6] J. Gilmore, R.H McKenzie, J. Phys. Chem. A, 112, 2162 (2008).

[7] K.M. Gaab, C.J. Bardeen, J. Chem. Phys. 121, 7813 (2004).

[8] H. Haken, G. Strobl, Z. Physik 262, 35 (1973).

[9] J.A. Leegwater, J. Phys. Chem. 100, 14403 (1996).

[10] P.W. Anderson, Phys. Rev. 109, 1492 (1958).

[11] P.W. Anderson, Rev. Mod. Phys. 50, 191 (1978).

[12] J.P. Keating, N. Linden, C.F. Matthews, A. Winter, Phys. Rev. A 76, 012315 (2007).

[13] A. Childs, E. Farhi, and S. Gutmann, Quant. Info. Proc. 1, 35 (2002).
[14] M. Mohseni, P. Rebentrost, S. Lloyd, A. Aspuru-Guzik, J. Chem. Phys. 129, 174106 (2008).

[15] P. Rebentrost, M. Mohseni, A. Aspuru-Guzik, arXiv:0806.4725 (2008).

[16] E. Rozbicki, P. Machnikowski, Phys. Rev. Lett. 100, 027401 (2008).

[17] S. A. Gurvitz, Phys. Rev. Lett. 85, 812 (2000).

[18] L. Gammaitoni, P. Hänggi, P. Jung, F. Marchesoni, Rev. Mod. Phys. 70, 223 (1998).

[19] D.E. Logan, P.G. Wolynes, Phys. Rev. B 36, 4135 (1987).

[20] D.E. Logan, P.G. Wolynes, J. Chem. Phys. 93, 4994 (1990).

[21] P. E. Parris and P. Phillips, J. Chem. Phys. 88, 3561 (1988).

[22] P. Phillips, Annu. Rev. Phys. Chem. 44, 115 (1993).

[23] L. Hartmann, W. Dür, and H. J. Briegel, New J. Phys. 9, 230 (2007).

[24] J. Cai, S. Popescu, and H. J. Briegel, arXiv:0809.4906 (2008).

[25] B. Misra, E. C. G. Sudarshan, J. Math. Phys. 18, 756 (1977).

[26] P. Facchi, H. Nakazato, and S. Pascazio, Phys. Rev. Lett. 86, 2699 (2001).

[27] N. Erez, G. Gordon, M. Nest, G. Kurizki, Nature 452, 724 
(2008).

[28] A. G. Kofman and G. Kurizki, Nature 405, 546 (2000).

[29] M. Cho, et al., J. Phys. Chem. B 10910542 (2005).

[30] A. Nazir, B.W. Lovett, S.D. Barrett, J.H. Reina, and G.A.D. Briggs, Phys. Rev. B 71, 045334 (2005).

[31] T.G. Owens, S.P. Webb, L. Mets, R.S. Alberte, and G.R. Fleming, Proc. Natl. Acad. Sci. USA 84, 1532 (1987).

[32] T. Ritz, S. Park, and K. Schulten, J. Phys. Chem. B 105, 8259 (2001).

[33] J. Adolphs, T. Renger, Biophys. J. 91, 2778 (2006).

[34] M.K. Şener, S. Park, D. Lu, A. Damjanović, T. Ritz, P. Fromme, and K. Schulten, J Chem. Phys. 120, 11183 (2004).

[35] O. Muelken, V. Bierbaum, A. Blumen, J. Chem. Phys. 124, 124905 (2006).

[36] A. Olaya-Castro, C. Fan Lee, F. Fassioli Olsen, and N. F. Johnson, Phys. Rev. B 78, 085115 (2008).

[37] S. Mukamel, Principles of Nonlinear Optical Spectroscopy, Oxford University Press, New York (1995).

[38] O. Mülken, A. Blumen, T. Amthor, C. Giese, M. Reetz-Lamour, and Matthias Weidemüller, Phys. Rev. Lett. 99, 090601 (2007).

[39] M.K. Henry, J. Emerson, R. Martinez, D.G. Cory, Physical Review A. 74, 062317 (2006).

[40] F. Müh, M. El-Amine Madjet, J. Adolphs, A. Abdurahman, B. Rabenstein, H. Ishikita, E.-W. Knapp, and T. Renger, Proc. Natl. Acad. Sci. USA 104, 16862 (2007).

[41] Y. Li, W. Zhou, R. E. Blankenship, and J. P. Allen, J. Mol. Biol. 271, 456 (1997).

[42] H. -P. Breuer and F. Petruccione, The Theory of Open Quantum Systems (Oxford Univerity Press, New York, 2002).

[43] D. Knuth. The Art of Computer Programming, Vol 1-3, Addison-Wesley (1997).

[44] Z. He, T. Ishizuka, and D. L. Jiangi, Polymer J. 39, 889 (2007).

[45] M. B. Plenio and S. F. Huelga, New J. Phys. 10, 113019 (2008); F. Caruso, A. W. Chin, A. Datta, S. F. Huelga, and M. B. Plenio, arXiv:0901.4454 (2009). 